\title{
The US CDC Global AIDS Program in China
}

\author{
Marc Bulterys
}

\begin{abstract}
The China-US Cooperation-Global AIDS Program (GAP) was a strategic technical collaboration program jointly implemented by the US Centers for Disease Control and Prevention and the Chinese Center for Disease Control and Prevention. This program developed, piloted, launched, and evaluated a broad range of projects supporting national and local HIV prevention and control programs; evidence-based decision-making; strengthening systems and capacity at national, provincial, and local levels; prioritizing high-risk geographic areas and populations; developing innovative approaches for scale-up; answering important scientific questions that can be most effectively answered in China but also with global implications for the HIV response; and increasing China's engagement with the global public health community and sharing critical lessons learned. A productive working relationship with well-conceived models, a results-based activity implementation plan, and proper linkage to the domestic policy process has made a significant contribution to HIV control and prevention in China.
\end{abstract}

\subsection{Historical Perspective of the HIV Technical Cooperation}

Infectious diseases remain a major cause of morbidity and mortality in China despite substantial public health progress in the past decades (Wang et al. 2008). Life expectancy at birth of the average Chinese person has increased from 35 years

\footnotetext{
M. Bulterys ( $\square)$

Global AIDS Program, U.S. Centers for Disease Control and Prevention, China Office, Beijing, China

Presently at Department of HIV and Hepatitis, World Health Organization, Geneva, Switzerland

e-mail: bulterysm@who.int
} 
in 1949 to 72 years in 2005 and 76.4 years in 2017 (World Health Organization, 2018), and infant mortality has dropped from 200 per 1000 live births in 1949 to 23 per 1000 in 2005 (Wang et al. 2008). These impressive gains were due in large part to a reduction in infectious disease mortality. However, infectious diseases remain an important public health concern in China even as the government implements major health system reforms to increase access to quality healthcare and respond to the increasing burden of noncommunicable diseases (Yang et al. 2008).

As an example, the severe acute respiratory syndrome (SARS) epidemic hit in 2003, bringing the country to a virtual standstill. Amid the frantic response to this epidemic, many questioned the readiness and openness of China to confront a newly emerging infectious disease epidemic (The Lancet 2003; Wang et al. 2008). At the same time, China's rapidly evolving HIV/AIDS epidemic called for a dramatic expansion of both prevention and treatment services (Bulterys et al. 2009; Kaufman and Jing 2002; Wu et al. 2001, 2007). Initially, HIV-1 infection was confined primarily to certain high-risk populations such as people who inject drugs (PWID) along drug-trafficking routes, and former commercial plasma donors (FPD) in rural communities in east-central China (Dou et al. 2010; Lu et al. 2008a; Wu et al. 1995, 2001; Yap et al. 2002). However, by 2008, official state media reported for the first time that HIV/AIDS had become China's leading cause of death among infectious diseases and that sexual transmission had become the primary mode of HIV transmission (Lu et al. 2008a; Wang et al. 2009).

In June 2002, the Secretary of Health of the US Department of Health and Human Services (DHHS) and the Minister of Health of China (the Ministry of Health is now known as the National Health Commission) signed a Memorandum of Understanding (MOU) entitled "China-US Cooperation on HIV/AIDS Prevention and Control." This MOU led to the establishment of the China-US Cooperation-Global AIDS Program (GAP), implemented jointly by the US Centers for Disease Control and Prevention (US CDC) and the Chinese Center for Disease Control and Prevention (China CDC). The bilateral cooperation program was officially launched in Beijing in March 2004. The launch of the program coincided with the nationwide scale-up of China's National Free Antiretroviral Therapy (ART) Program (Zhang et al. 2007, 2009), which was originally initiated in 2002 in response to the significant iatrogenic epidemic in central China (Dou et al. 2010; Zhang et al. 2008a, b).

The principal implementation and coordination agency of this program was the China CDC under the leadership of the Chinese Ministry of Health (now National Health Commission). In 2006, the US-China collaboration was integrated into the President's Emergency Plan for AIDS Relief (PEPFAR), which provides an overarching framework for the US government's response to the global HIV/AIDS epidemic and remains the largest commitment by any nation to respond to a historic global health crisis (Fauci and Eisinger 2018). Under the PEPFAR umbrella, US-China collaboration on HIV/AIDS was expanded to include collaboration with the US Agency for International Development (USAID) in Yunnan province and Guangxi Zhuang Autonomous Region (hereafter Guangxi). The initial PEPFAR budget for activities in China for fiscal year 2006 was 9.8 million USD, with a funding peak of 10.3 million USD in fiscal year 2009. Although USAID ended its China-specific programming as 
of 2012, strategic technical collaboration between the China CDC and the US CDC through the GAP has remained active until the present time.

The GAP has been an integral part of broader US-China cooperation on health, which has also included collaboration with the US National Institutes of Health through multiple research grants. Collaboration between the two countries' CDCs also expanded to include other communicable and noncommunicable diseases. The US CDC maintains a presence in China with several American staff assigned to the US Embassy in Beijing as technical advisors, as well as approximately 30 locally hired staff in the time period between 2010-2015 providing technical, program management, and administrative support to the collaboration. Staff focused on HIV/ AIDS represented approximately one third of this complement of China-based experts, and the capabilities of these staff are complemented by US CDC Atlantabased technical experts who travel to China to provide assistance in specific technical areas as requested by the Chinese government.

Within this historical, epidemiologic, and programmatic context, the US CDC has supported China in carrying out strategic activities in HIV surveillance, prevention, care, and treatment and other areas. These activities have been fully integrated into the Chinese national response and have led to measurable achievements within China's comprehensive HIV control and prevention program (Li et al. 2013; Lin et al. 2012; Wu et al. 2011). The collaboration continues to evolve and develop as the HIV epidemic in China and globally evolves as well. This chapter aims to review and highlight some of the progress made, with particular focus on the first decade of the bilateral HIV technical cooperation between the US and China CDCs, and to share relevant experiences in this dynamic context.

\subsubsection{Programmatic Approach to Collaboration}

Over its history, the joint vision of the US-China cooperation has been to assist the government of China to reduce HIV transmission and mitigate the impact of AIDS, within the context of specific national goals. For 2015, these national goals included maintaining the overall number of HIV cases to less than 1.2 million, reducing HIV incidence by $25 \%$, and reducing mortality by $30 \%$. These goals were to be achieved by ensuring that (a) $90 \%$ of those who were members of high-risk groups had access to effective prevention measures, (b) $70 \%$ of people living with HIV (PLWH) knew their status, (c) greater than $80 \%$ of those who were eligible for ART were receiving this lifesaving treatment, and (d) prevention of mother-to-child transmission (PMTCT) exceeded $80 \%$ coverage nationwide (Wu et al. 2011).

As the HIV epidemic and the national response evolved, the goals of the GAP collaboration evolved as well, although fundamentally the approach has always focused on epidemic control. Because China's HIV epidemic has been concentrated geographically in seven provinces, in addition to support at the national level, the GAP has maintained a technical and incentive-based collaboration with provincial-level health authorities based on need and potential for epidemic impact.

Over the history of the China-US collaboration, the provincial and programmatic focus has included a total of 15 provinces. Anhui and Henan provinces were at the 
center of the iatrogenic epidemic driven by commercial plasma donation among rural farming communities in the early 1990s, and in these provinces, there has been a key emphasis on expanding access to, and improving the quality of, HIV treatment and care services. In Guangxi, Guizhou, Guangdong, and Yunnan provinces as well as Xinjiang Uyghur Autonomous Region (hereafter Xinjiang), where the epidemic was initially driven by injecting drug use, the key emphasis has been on counseling and testing, as well as active case-finding and targeted interventions, including harm reduction capacity-building and follow-up services for PLWH (Li et al. 2013). Beijing municipality, Heilongjiang, Jiangsu, and Shandong provinces, Inner Mongolia Autonomous Region (Inner Mongolia), Ningxia Hui Autonomous Region (Ningxia), and Tibet Autonomous Region (Tibet) have concentrated epidemics among key populations, particularly among men who have sex with men (MSM; Wu et al. 2013). In these provinces, the primary emphasis has been on studying the epidemic among key populations and supporting innovative, targeted prevention, care, and treatment activities and capacity building among the local implementing units. As the collaboration has progressed, direct support has increasingly focused on those provinces and prefectures with the highest HIV prevalence, and by 2014, the focus centered on Guangdong, Guangxi, Guizhou, Hunan, Yunnan, Xinjiang, and Sichuan's Liangshan prefecture.

GAP output-driven provincial collaborations have focused on ensuring that all cases found via HIV/AIDS surveillance, voluntary counseling and testing (VCT), provider-initiated testing and counseling (PITC), and other means received timely notification, follow-up, and referral services. As a result, all GAP-supported provinces have established sound and smooth linkage programs for case finding and case management. For instance, the CD4 testing rate among PLWH, the percentage of eligible HIV/AIDS patients on ART, and the viral load testing rate of patients on ART in the 15 GAP-supported provinces dramatically increased from $30 \%, 48 \%$, and $27 \%$ in 2008 to $71 \%, 90 \%$, and $80 \%$ in 2011 , respectively. This is an overall measure of success for the collaboration and shows the productivity of this longterm engagement. A public health partnership has been built on a foundation of mutual trust, commitment, and dedication to shared goals.

\subsubsection{Surveillance, Public Health and Clinical Systems, and Capacity Development}

In response to key challenges within the national HIV/AIDS control and prevention program, GAP adopted a classical public health approach to its early strategic engagement in China. The strategy aimed to decrease the number of new infections by (a) targeting disease surveillance to enable evidence-based public health programming and decision-making, (b) linking PLWH to prevention support services to prevent secondary transmission, and (c) improving access to, and quality of, care and treatment services.

Specifically, GAP supported the establishment or strengthening of 649 national or provincial sentinel surveillance sites in 15 provinces and further ensured that these 
sites were actively providing linkages for those individuals who were found to have HIV infection. As a result, the number of sentinel surveillance sites increased sixfold during the period from 2003 to 2008, and 18,820 PLWH received diagnoses at these sites. This activity increased the coverage and quality of surveillance information available for decision-making, while also increasing local capacity for developing referral systems, care linkages, and case management capacity (Wang et al. 2010). Over the subsequent years, these surveillance sites have been transitioned to local public health authorities and continue to function as an important tool for both local and national public health decision-making. GAP has also supported an external evaluation of the adequacy of the HIV surveillance system in China (Lin et al. 2012), and the findings of this evaluation have been used by national authorities for further system strengthening (see Chap. 2 for more information).

As the numbers of PLWH increased, there was a compelling public health need to establish a systematic approach that linked these individuals to appropriate follow-up testing and care and the prevention of secondary transmission. Again, the national program called for implementation of a public health approach based on increased rates of case finding, epidemiological investigation, CD4 testing, and follow-up services. As part of the China-US collaboration, pilot programs were established in Yunnan and Guangxi to increase the proportion of individuals who knew their status and increase the number of PLWH who completed at least two linked follow-up visits for care and support. These pilot efforts were carried out from 2006 to 2011, and the epidemiological investigation rate rose from $25 \%$ to 99\% in Yunnan and 31\% to 99\% in Guangxi. Since 2009, these measures have served as national core indicators for all provinces, with similar improvements over time- a key contributor to program success at the national level (see Chap. 23 for more information).

As systematic public health efforts to identify PLWH and link them to appropriate services intensified, certain gaps within the clinical care systems also emerged. Particularly in the rural areas hardest hit by the iatrogenic, plasma donation-related epidemic of the 1990s, it became apparent that the healthcare providers did not have adequate training or clinical mentoring to provide quality HIV care and treatment to increasing numbers of patients on ART. In close collaboration with the NCAIDS Division of Treatment and Care at China CDC and the Clinton Foundation, the US CDC established China's first rural AIDS Clinical Training Center in 2004 in Lixin county, Anhui province. Its mission was to provide in-service AIDS clinical training to rural clinicians selected from local Anhui and other high epidemic provinces in China. These clinicians had little training or experience dealing with patients on ART, and the Center's intensive 10-week residential training program included classroom training, direct clinical mentoring, and outreach within the local area to township and local hospitals. This approach proved extremely successful. The model was replicated in two additional provinces in the later years of the GAP collaboration, and in 2011, leadership for the training center in Anhui was transitioned to the provincial government (see Chap. 27 for more information).

GAP also introduced state-of-the-art surveillance methodology to increase case finding. The program supported the drafting of the Protocol for Respondent Driven 
Sampling (RDS) Surveys for Integrated Biological and Behavioral HIV Surveillance in MSM in China (Wu et al. 2013). Prior to protocol drafting, GAP supported pilots in Beijing, Shandong, Guangdong, and Ningxia using RDS to conduct surveys among MSM, female commercial sex workers (FSW), and PWID. Through these pilots, stakeholders developed a better understanding of field implementation methods, which proved invaluable in the national adoption of RDS. GAP supported timelocation sampling among MSM in Shenzhen. This sampling method was introduced internationally and had never been applied in China (see Chap. 2 for more information).

An additional priority during this period was developing successful models for PMTCT of HIV, and the China-US collaboration expanded to include work within the maternal and child healthcare system. GAP helped to demonstrate that with conservative cost estimates, integrated nationwide HIV and syphilis testing of pregnant women in prenatal care in China was substantially more cost-effective than HIV screening alone (Owusu-Edusei et al. 2014). Comprehensive, integrated PMTCT efforts were highly effective in reducing mother-to-child HIV transmission to very low levels whether in rural or urban settings (Shan et al. 2014; Song et al. 2013). In 2007, GAP started to collaborate with the Guangxi Women and Community Health Bureau and the Guangxi Maternal and Child Health Hospital to pilot an integrated PMTCT program in 112 antenatal care clinics (ANCs). By fostering integration across China's three-tier health system, ANC, and ART services, this program successfully linked women with HIV infection and their children to HIV care and treatment. It was shown to be an effective model that was expanded first to the entirety of Guangxi where the approach continues to deliver results. From 2009 to 2011, 174,776 pregnant women (97\%) were tested for HIV, with $728(0.41 \%)$ diagnosed with HIV infection. HIV testing coverage during pregnancy significantly increased from $80 \%$ in 2009 to $87 \%$ in 2010 and to $91 \%$ in 2011 . From 2009 to 2010, 12 out of 379 infants born to HIV-infected women tested HIV-positive, for a transmission rate of $3.2 \%$. In part thanks to these early results, the National Center for Women and Children's Health (NCWCH) expanded coverage and implemented this program in all seven of the highest prevalence provinces in China. By 2015, the comprehensive, integrated PMTCT program was implemented nationwide by the Chinese government (see Chap. 15 for more information).

While the GAP remained closely integrated with the national response, the flexibility of the international cooperation model also allowed for some experimentation, and as a result, a number of innovative approaches were tested within the specific Chinese context. From an initial emphasis on controlling the iatrogenic epidemic, the Chinese response shifted quickly to addressing the challenge of injecting drug use, as a major contributor to the Chinese HIV/AIDS epidemic in the early years of the US-China collaboration (Wu et al. 2015).

Drug use has been an important driver of the HIV/AIDS epidemic in China. In 2003, there were 1.03 million registered drug users in China, $80 \%$ of whom were PWID (Wang et al. 2009; Wu et al. 2007). In 2003, 44\% of PLWH in China contracted HIV via injecting drug use. To slow the dual epidemics of HIV and drug use, a methadone maintenance treatment (MMT) program was piloted in 2004 with 
eight clinics in five provinces serving approximately 1000 clients (Lu et al. 2008b). Since then, the National MMT Program has scaled up rapidly. By the end of 2011, the National MMT Program was comprised of 738 clinics serving more than 340,000 clients cumulatively (Li et al. 2013; Liu et al. 2013). However, China faced a challenge in delivering methadone to PWID living in the rural and often remote border areas where the drug trade flourished.

In an innovative effort to address this challenge, in 2006, GAP supported the establishment of the first mobile MMT clinic in China in Dehong prefecture, Yunnan province, an area with high HIV prevalence and incidence at the border with Myanmar (Duan et al. 2010). The mobile unit covered 50 villages, and not only expanded coverage but also increased accessibility and maintenance on therapy reached $76 \%$. As of the end of 2015, there were 785 full-service "brick-and-mortar" MMT clinics as well as 29 MMT mobile vans operating across China. These "sites" also supported a further 325 satellite MMT sites. Since 2011, this expanded network has been providing services to roughly 200,000 heroin users each year (Wu and Pisani 2017) (see Chap. 9 for more information).

\subsubsection{Key Populations, Laboratory System Development, and Implementation Science}

As the collaboration strengthened and deepened, it also adapted to respond to the evolving characteristics of the HIV epidemic in China and the needs of the national program. With a strong national surveillance system in place to guide program and policy decisions, the concentration of the epidemic among key populations became very apparent. While injecting drug use remained a key factor in HIV transmission, by 2008, sexual transmission had become the primary mode of HIV transmission (Wang et al. 2010). This necessitated new approaches to reach additional at-risk populations - not only PWID but also commercial male and female sex workers (and their clients) and MSM. As these groups are often vulnerable and stigmatized, interventions to address their public health needs must be carefully considered. GAP led the way in developing evidence-based approaches to these challenging issues.

\subsubsection{Drug Users}

The rapid scale-up of MMT has benefited thousands of drug users with decreased drug use and criminality, increased quality of life, and higher rates of employment (Li et al. 2013; Wu and Pisani 2017). However, one of the main challenges in the early years was the very high dropout rate. A relatively low dosage of methadone may have been a key reason behind this (average dose was only $48 \mathrm{mg}$ in 2007), as well as low training coverage and high turnover among MMT providers. Although it has been shown in other settings that higher methadone doses can lead to higher retention and other favorable MMT outcomes, it is often not practiced in the field. In 2010, GAP received funding through PEPFAR to support the China CDC, Guangdong CDC, Guangxi CDC, and Guizhou CDC in a joint public health 
evaluation (PHE) entitled "A Methadone Maintenance Treatment Outcome Study in Three Provinces in China. Comparative Evaluation of the Impact of an Intensive Health Care Provider Training Program Combined with Expanded Services on Treatment Retention, Heroin Use, Methadone Dosing and HIV Risk Practices."

The purpose of this study was to evaluate the impact of an intensive healthcare provider training program combined with expanded services targeting improving treatment retention, methadone dosing, and heroin use. The study aimed to determine reasons behind current dosing practices as well as reasons clients remain in and drop out of MMT. The study also aimed to evaluate the effectiveness of a tailored education program for MMT service providers via measurement of methadone dose levels prescribed to new patients. The effects of methadone dose, with and without the inclusion of additional psychosocial services, were measured as MMT retention and illicit opioid use. This study consisted of a qualitative, formative assessment followed by a three-arm cluster-randomized controlled trial. The qualitative study using key informant interviews was conducted on a sample of MMT clinic staff, clients, family members of clients, and opiate dependent persons in the community during 2011. The findings were presented at the 19th International AIDS Conference in Washington DC (Han et al. 2012).

At the end of 2011, the randomized community intervention trial was officially launched, and the first round of training was completed in three provinces. The trial was conducted in 54 MMT clinics, which were randomized into one of three study arms: arm 1 is control group (standard of care); arm 2 gives intensive healthcare provider training on prescribing methadone dosage; arm 3 gives intensive healthcare provider training on prescribing methadone dosage, plus onsite psychosocial counseling services and enhanced peer support to clients. The study has been enrolling patients in Guangdong, Guangxi, and Guizhou provinces for several years, and 12-month follow-up of all participants has been completed in 2017. Findings from the trial have been used by the national MMT program to seek further service delivery improvements, and the findings will be published soon in the peer-reviewed literature.

The challenges described here are not unique to China's MMT program-lessons learned may offer valuable guidance to harm reduction programs being developed in many other countries (Li et al. 2013; Wu et al. 2011). Lessons learned during the implementation of this study include the critical importance of implementation science in program quality improvement as well as the crucial role that training programs play in MMT clinic service quality and client outcomes improvement (see Chaps. 9 and 27 for more information).

\subsubsection{Female Sex Workers (FSW)}

Although commercial sex work is illegal in China, there are an enormous number of FSW working in China with estimates ranging from two to ten million (Flannery 2017; Lu et al. 2006; Pirkle et al. 2007; Shen and Csete 2017). Commercial sex work remains highly stigmatized in China and differs from other Asian countries. Fewer sex workers work in brothels, and as a result, epidemiologic studies are harder to conduct, and therefore, data about their health experiences remain 
relatively sparse. A review published by staff associated with the GAP collaboration shows relatively high median rates of sexually transmitted infections (STIs) but a stable and relatively low rate of HIV (prevalence ranged from $0 \%$ to $10.3 \%$ in different settings, median 0.6\%; Poon et al. 2011).

Because of the potential impact of this relatively hidden population, the GAP conducted intervention activities such as advocacy, training, peer education, and promotion of condoms. It also promoted friendly STI services, improved VCT services, HIV testing, care seeking at STI, gynecology, and tuberculosis (TB) clinics. The program also provided test results notification and counseling, as well as follow-up and referral services. In addition, GAP supported an intervention model pilot study focused on older male clients and low-fee FSW in Hunan and Guangxi. These pilot interventions provided information on the local epidemic and scientific evidence for further intervention strategies. GAP also conducted comprehensive HIV control and prevention among FSW working in China's border regions with Myanmar and Vietnam, with the goal of implementing a "treatment-as-prevention" (TasP) strategy.

Although the prevalence of HIV among FSW in China is relatively stable and below $1 \%$ overall at the national level, the prevalence of STIs appears to be increasing (Han et al. 2010; Poon et al. 2011; Wang et al. 2010). Studies have shown STIs that cause ulcers or inflammation may facilitate increased transmission efficiency of HIV and other STIs. Moreover, many HIV/STI prevalence studies, including surveillance efforts in China, are focused primarily on establishment-based FSW, which underestimates the true prevalence of HIV, other STIs, and high-risk behaviors among FSW (Lin et al. 2012). Studies have shown that low-tier FSW (i.e., FSW who are street-based and charge low fees) have higher rates of HIV and syphilis and lower rates of condom use (Chen et al. 2012, 2016).

With GAP financial and technical support, a survey was conducted in 2012 in five cities in Guangdong, Guizhou, and Shandong provinces and completed by the end of 2012. The primary objective of the survey was to better understand risk perceptions and behaviors of low- to medium-fee FSW in China as well as seroprevalence rates of HIV, syphilis, and herpes simplex virus type 2 (HSV-2) in this population. A total of 1487 eligible low-fee and medium-fee FSW were recruited in the study. Findings have been published in close collaboration with NCAIDS and show that low-fee FSW were more likely to have HSV-2 infection, but not more likely to have HIV or syphilis infection, compared with medium-fee FSW. The survey also demonstrated that low-fee FSW in China have unique risks for acquiring STIs, in part due to greater economic pressures (Han et al. 2016). Tailored interventions targeting low-fee FSW, in particular, that incorporate prevailing condom use negotiation challenges are urgently needed. The potential for prevention methods alternative to male condoms has received greater attention in recent years including pre-exposure prophylaxis (PrEP), post-exposure prophylaxis (PEP), and female condoms. Gaining knowledge on awareness and acceptability of these prevention methods among high-risk FSW in China will help inform the potential implementation of these prevention methods in China (see Chaps. 3 and 7 for more information). 


\subsubsection{Men Who Have Sex with Men (MSM)}

In 2008, China launched a major initiative to address HIV/AIDS among MSM, including strong promotion of condom use, expanded prevention programs, increased counseling and testing, and improved access to treatment. The GAP mobilized to support this national effort. A number of the innovative approaches initially piloted with the support from GAP have become models for scale-up at the local, provincial, or national levels.

GAP recognized the critical role that communities and opinion leaders play in engaging with this vulnerable population and therefore supported a popular opinion leader (POL) intervention model. This model first started as a pilot program in Mianyang, Sichuan province, and expanded to Guizhou province. It is now a nationwide program and is the standard intervention model under the "National MSM Population Comprehensive Prevention and Control Pilots." The program expanded case follow-up mechanisms to include MSM peer groups.

Counseling and testing was critical to the success of the national initiative, so GAP piloted a number of new strategies, including a web-based VCT program in Heilongjiang province that included linked STI and HIV care services. This successful model was later scaled up by the Global Fund to Fight AIDS, Tuberculosis and Malaria in China. In addition, GAP led the way in piloting HIV rapid testing as a way to increase testing rates among MSM. Pilot programs in Jinan, Kunming, Mianyang, Harbin, Qiqihaer, Jiamusi, Daqing, and Mudanjiang suggested that this was a feasible and effective model for identifying and managing MSM with HIV infection. Similarly, a rapid HIV testing pilot in a gay bathhouse in Tianjin demonstrated this method's value for identifying many HIV cases among MSM in hard-to-reach places. This pilot was selected by China in 2011 as a model for best practices.

GAP also introduced the concept of couples counseling and testing within the MSM population in China. Working with MSM couples is a new challenge for counselors and requires different skills and approaches than traditional couples counseling. To ensure that counselors had these needed skills, GAP sponsored a training workshop, which was held in Chengdu, and collaborated on the development of a couples testing and counseling manual specifically for MSM. The results of a pilot showed that the new model was well accepted by MSM. The model was then replicated in Xinjiang and Beijing and then expanded further to other provinces.

In addition, GAP collaborated with the largest gay male dating website in China (www.bf99.com) and NCAIDS to analyze migration patterns of MSM in China. The dating website contained data on nearly 800,000 eligible MSM, of which 35\% were migrants. De-identified data showed that MSM migration from southwestern China, which has the highest HIV prevalence in this population, to coastal cities with lower prevalence has implications for the spread of the HIV epidemic as well as the need for HIV care services (Mi et al. 2016).

GAP also collaborated with NCAIDS on a large-scale, national 61-city survey of HIV prevalence and risk behavior in MSM (Wu et al. 2013). These early efforts have paved the way for greater and deeper collaboration between the USA and China in order to reduce rates of HIV among MSM-a continuing challenge for both countries and for many other countries (see Chap. 8 for more information). 


\subsubsection{Treatment and Care}

Since 2008, the program has provided technical assistance for data analysis and utilization of the National Free ART Program (NFATP) in order to improve patient outcomes (Cheng et al. 2015; Dou et al. 2010; Ma et al. 2010; Zhang et al. 2009, 2011, 2014; Zhu et al. 2014). It also supported implementation of the basic care package of services to PLWH such as VCT, condom use promotion, CD4 testing, opportunistic infection (OI) prophylaxis, TB/HIV coinfection treatment, ART, PMTCT, STI referral and relevant laboratory testing, medication adherence counseling, and PLWH selfhelp organizations. GAP also helped NCAIDS to conduct community advocacy, VCT, outreach, intervention, and advocacy events; explore care models that involved community-based organizations (CBOs) and nongovernmental organizations (NGOs); improve the case management system; support pilots to increase adherence and improve and expand treatment quality and coverage; improve coinfection treatment and management; and actively promote ART based in MMT settings.

GAP supported the Treatment and Care Division of NCAIDS to develop the first version of the National Free ART Manual (including Guidelines for Using Cotrimoxazole for the Prophylactic Treatment of Opportunistic Infections) and the National Guidelines for Second-line ARV treatment in 2008. Subsequently, GAP supported the second and third revisions of the National Free ART Manual, drawing from best practices of GAP-supported pilot programs, and supported the development and distribution of CDs of "Life Saving Drugs, Never Stop," Treatment and Care Service Handbook, and ARV pillboxes to improve adherence and patient follow-up services. GAP also supported NCAIDS and the National TB Center to develop the Implementing Guideline for HIV and TB Co-infection Pilots and the National Diagnosis and Treatment Guideline for HIV and TB Co-infection. The National TB Center and Global Fund Round 5 HIV/TB coinfection pilot program in six sites across four provinces was expanded to 134 counties. Technical support for data analysis and utilization was provided to TB/HIV programs. GAP also helped the National TB Center to conduct pilot programs for the use of isoniazid preventive treatment (IPT) among HIV/AIDS patients in Sichuan and Anhui.

In addition, pilot programs on community-based care service to improve treatment adherence, treatment service quality, treatment coverage, and coinfection treatment and management according to local situations were supported. This included cell phone adherence reminders for patients in Anhui; operational support to 15 village-level medical units in the most severely affected area in Anhui; treatment and care programs in Heihe, Qitaihe, and Boli in Heilongjiang; TasP among discordant couples in Guangxi and Xinjiang; and the HIV/TB, HIV/HCV/HBV coinfection programs in Hunan and Sichuan.

Critically, GAP supported NCAIDS, China CDC in their continuous improvement efforts for the national treatment information system, which was upgraded at the end of 2010. This completed the computerization of medical records and data reporting systems in all ART treatment sites, which greatly improved data quality and data reporting efficiency. Management and coordination of ART treatment at central and peripheral levels were also enhanced. Annual national data quality checks have been conducted beginning in 2009. 
In 2011, US CDC experts collaborated with NCAIDS experts to analyze and evaluate overall antiretroviral drug resistance data, reported resistance sequencing quality, and reliability of resistance test results from national and provincial drug resistance laboratories. In addition, the level of ARV drug resistance in the Chinese population was analyzed, and development of a report on resistance was discussed. These activities had a significant impact on HIV drug resistance work in China (see Chaps. 4 and 13 for more information).

\subsubsection{Rural Populations and Ethnic Minorities}

As mentioned previously, GAP collaborated with the Clinton Foundation, China CDC, and the Anhui Provincial CDC to establish a rural clinical training center in Lixin County, and by 2011, that training center was transferred to the local health authority. Anhui's iatrogenic epidemic was under reasonable control, but the center continues to function as it provides an excellent opportunity for clinicians to gain familiarity with second-line treatment regimens as many of the patients on treatment there were diagnosed early in China's HIV epidemic.

However, new HIV infections continue to occur in large numbers in China's rural areas, especially in the southwest and among China's ethnic minority populations (Zhang et al. 2013). Although less than $10 \%$ of China's population belong to one of the 55 recognized ethnic minority groups in China, people with minority affiliation have a higher burden of HIV infection on aggregate (i.e., when all minorities are lumped together and compared to Han Chinese despite each individual minority group having greater or lesser odds of HIV when taken alone; Pan et al. 2016). Therefore, in 2010, GAP established a second rural clinical training center in Luzhai county, Guangxi, an area with a significant heterosexual and drug use-driven HIV epidemic. Then in 2012, collaborating with Merck Foundation, local CDC authorities, and NCAIDS, GAP established the Zhaojue training center in Zhaojue county, Sichuan province. This hard-hit area had a primarily injecting drug use-driven HIV epidemic among the Yi ethnic minority group. The training facility in Zhaojue (funded by GAP) provided ongoing mentoring to 20 local clinicians and helped treat over 200 additional PLWH from the Yi community between 2013 and 2014.

These rural clinical training centers continue to make significant contributions to the national HIV/AIDS response and complement the national urban training centers. By 2014, 259 graduates completed the rural HIV/AIDS clinical training program, 111 of them from Lixin center, 112 from Luzhai center, and 36 from Zhaojue center. Dual referral systems were established between county- and township-level health facilities at the Lixin and Luzhai county health systems, as part of the training activities. All rural clinical training centers have been transferred to the local health authority. Results of an evaluation conducted among graduates who attended a refresher training in December 2011 showed that $96 \%$ of them were still providing HIV/AIDS care and treatment; $80 \%$ of them became local AIDS clinical experts; and collectively, these trainees provided treatment and care services to at least 30,000 HIV/AIDS patients in China, many in remote or mountainous areas. 
In order to meet the increasing need for quality, comprehensive HIV clinical care, GAP worked with I-TECH (International Training and Education Center for Health, University of Washington) in 2013 to design and develop a 2-week training curriculum on HIV/AIDS care for nurses working in rural areas of Liangshan prefecture in Sichuan province. This program is being implemented by Liangshan Infectious Diseases Hospital to cover all nurses working on HIV/AIDS treatment and clinical care across the entire prefecture. Merck Foundation, in cooperation with NCAIDS, continues to provide financial support for this program. This curriculum is intended to fill the gap in training for nurses of the national training program on HIV/AIDS treatment and care. Therefore, NCAIDS has scaled up this essential training for nurses to all 12 national training centers.

\subsubsection{Strengthening the National HIV Laboratory System}

In order to ensure an effective national HIV/AIDS response, a country's laboratory system must be strengthened. Since its inception, the GAP has focused on capacity building at the National HIV Reference Laboratory (NHRL). Goals included improving HIV/AIDS testing laboratory guidelines and operating procedures, expanding the number of HIV testing laboratories in China, introducing novel testing technologies, and strengthening the quality control system of the national laboratory network (Jiang et al. 2010). This has been a very productive area for collaboration, especially in the period from 2008 to 2013. GAP has helped NHRL as well as provincial and local laboratories to make remarkable progress.

A key area of support has been technical guideline development and national and provincial capacity and testing quality improvement, beginning with the National Guidelines for HIV Detection in 2004 and again in 2009, but also national guidelines for CD4+ $\mathrm{T}$ Lymphocyte Enumeration and Quality Assurance, HIV-1 Viral Load Measurement and Quality Assurance, Hepatitis C Virus (HCV) Detection (2012), HIV-1 Rapid Tests, Drug Resistance Quality Assurance, and HIV Proficiency Testing. In addition, GAP supported the development of a training manual for early infant diagnosis (EID) technology and an HIV incidence testing protocol for use in 15 provinces.

In addition, GAP strengthened the capacity of NHRL through digitization and proficiency testing of an electronic report management system (v1.0, v2.0, v3.0, and v4.0) including an administration software system, workstations, and client software systems and assisted with internal quality control (QC) panels for HIV and HCV antibody testing and preparation of syphilis antibodies. GAP support enabled NHRL to expand proficiency testing (PT) activities nationwide and increase the national PT panel distribution from one to three times per year, as well as launch PT programs for antibody, CD4, viral load detection, and HIV-1 BED incidence testing (Jiang et al. 2010). Overall, the number of laboratories that participate in PT programs increased from 57 to 370 .

In addition, the program assisted NHRL to annually monitor the quality of domestic and international HIV testing reagents available in the Chinese commercial market in order to assure national testing quality. Finally, and perhaps most significantly, GAP assisted NHRL to improve management and to obtain the 
highest international laboratory accreditation offered by the American Society of Pathologists in 2011.

GAP provided support that built capacity below the national level as well, by supporting trainings and workshops to strengthen the capacity of local laboratory staff, including national training courses on PT and the electronic reporting and management system; national training courses and workshops on HIV-1 incident infection detection, surveillance, and data analysis; conferences on the PT program; a workshop on alternative HIV testing algorithm; a conference on laboratory HCV testing and quality assurance; and a conference on HCV testing reagent evaluation. Virtually all of the 15 GAP-supported provinces received some support and only a few examples are provided here.

With GAP support, Anhui held technical training for laboratory personnel, organized QC activities, strengthened supervision and inspection of the laboratory, and established a set of reasonable quality assessments for the HIV testing laboratory system. Every year, the province trained laboratory personnel and assessed HIV laboratories. The project accelerated laboratory establishment and standardization of work. In Beijing, with support from GAP, the laboratory network's capacity improved significantly. Technical skills for performing screening tests and HIV diagnosis technologies advanced dramatically through years of persistent training and evaluation. HIV infection was diagnosed in a timely manner, case finding was accurate, and prevention strategies were well developed. In Guangdong, GAP's efforts strengthened QC of HIV screening and confirmatory laboratories in the province and established a monitoring system for ARV drug resistance. A monitoring system for HIV incidence among high-risk groups was also established to evaluate the impact of the HIV/AIDS response. GAP supported Guangxi CDC to implement various advanced laboratory methods. In Heilongjiang, at the end of 2011, a laboratory detection network was established. The network was composed of one confirmation central laboratory, four confirmation laboratories, nine HIV screening central laboratories, 427 HIV screening laboratories, and 146 detection points. This ensured the network laboratory QC was standardized in the entire province.

NHRL's capacity to engage in innovation and to explore the potential of new laboratory technology made the GAP collaboration fertile ground for exciting new areas of collaboration. The work done in collaboration with China has the potential to significantly and positively contribute to the global response to HIV-not only to the Chinese national response. Some of the areas where collaboration was initiated with this goal in mind include evaluation of new point-of-care (POC) CD4 analyzers (Liang et al. 2015), development of dried blood spot (DBS)-based HIV-1 drug resistance testing, HIV-1 incidence assay including BED and limited antigen assays (Duan et al. 2010), use of RT-PCR on pooled specimens, establishment of molecular assays for HIV detection including DBS-based EID tests, alternative strategies of HIV antibody testing, HIV-2 detection, molecular epidemiologic investigation techniques of HIV infections, evaluation of HCV detection reagents and testing strategy assessment, and development of molecular testing and HCV genotyping (see Chap. 5 for more information). 


\subsubsection{Other Innovative Activities and Implementation Science Research}

The GAP also provided technical support to innovative activities in the provinces and at national divisions at NCAIDS, including an MSM cohort study in Ningxia; a survey of MSM risk behaviors in Harbin; a survey of amphetamine use in different risk groups in Shandong; an MSM HIV-1 molecular epidemiological study; an MSM survey in Lhasa, Tibet; an exploration of comprehensive intervention models involving community health clinics and self-help organizations for key populations; psychological intervention on behavior change among HIV-infected MSM in Beijing; a study of HIV transmission risk, impact factors, and responses between couples in Yunnan, Henan, Sichuan, Guangxi, and Chongqing; a social network study of individuals newly diagnosed with HIV infection; a size estimation of FSW, drug users, and MSM; a cost analysis of cotrimoxazole's prophylaxis effect on reducing mortality; a survey of women from high epidemic areas marrying rural farmers in Shandong; and a pilot study of couples/partner notification.

These innovative activities, in close technical cooperation with NCAIDS, have provided scientific evidence and established operational mechanisms to improve the effectiveness of the HIV/AIDS response at provincial and national levels. After a decade of technical cooperation, the program has been successfully integrated into the national and provincial HIV/AIDS programs (Wu et al. 2011). These innovative pilot projects, with close linkage to the policy process, have made valuable contributions and helped generate better data to guide the development of more targeted and responsive national HIV/AIDS policies. Some of the pilots and models that the program successfully implemented have been promoted and replicated nationwide.

\subsubsection{HIV PITC in Luzhai County, a High Epidemic Area}

Luzhai county (population 413,200) is comprised of four towns and five villages and is located in the central northern region of Guangxi. There are five county health facilities, ten township (village) health clinics, and four screening test laboratories (i.e., at the County CDC, the County People's Hospital, the Chinese Traditional Medicine Hospital, and the County Women and Children's Hospital). All medical facilities in the county are capable of delivering related HIV counseling and rapid testing. There was also one MMT clinic, one extended MMT clinic, one ART site, and one extended ART site.

The first HIV-infected patient in Luzhai county was reported in 1998. By the end of 2010, the cumulative number of HIV/AIDS cases reported in Luzhai was 3241 (1411 of whom had already developed AIDS), and the cumulative total number of HIV/AIDS-related deaths reported was 1003. Overall adult HIV prevalence in the county was close to $1.0 \%$. In 2011, there were a total of 956 AIDS patients who received ART in Luzhai County People's Hospital with 209 new enrollments. Objectives were to simplify routine HIV testing in the clinical setting, find more HIV cases as early as possible, and link PLWH to prevention, treatment, and care services to prevent secondary sexual transmission. With the support and technical 
assistance from GAP, county government decided to include routine HIV testing into the government performance review system and included it in the annual review for all township and village leaders.

With GAP support, 86,569 HIV screening tests were performed in 2011 (accounting for $19 \%$ of the population of Luzhai), and 1174 new HIV-infected persons were found. HIV screening increased by nearly $300 \%$ and the number of cases found increased by $135 \%$ compared to 2010 .

\subsubsection{Couples HIV Testing and Counseling Among MSM in Chengdu}

Originally developed and promoted by US CDC, couples HIV testing and counseling (CHTC) has been shown to be an effective strategy among heterosexual couples. As MSM couples differ from heterosexual couples, scaling up CHTC for MSM couples required piloting in China and careful evaluation. A pilot program was designed, in collaboration with faculty from Emory University, to examine the feasibility and effectiveness of CHCT among MSM couples. It aimed to train $30 \mathrm{MSM}$ counselors, develop MSM-friendly promotional leaflets and posters on CHTC, and develop a CHTC working manual targeting MSM couples.

A workshop on CHTC for MSM couples was held in Chengdu in March 2011. A total of 36 trainees participated in this workshop, including staff from MSM CBOs, local CDCs, and community volunteers. Training covered the principles, procedure, and skills for CHTC. The CHTC site was developed with the Chengdu Gay Care Organization (CGCO). A program manager was appointed to oversee monitoring, QC, and data management. Four experienced counselors were chosen and trained to provide CHTC. Several doctors serving as volunteers were responsible for conducting HIV testing.

Multiple community mobilization strategies were undertaken to raise awareness of CHTC for MSM couples. CHCT was promoted in gay venues through social networking and gay websites. An electronic version of CHCT message was designed and disseminated in "QQ" instant messenger groups and chat rooms on the Internet. In total, 53 couples (106 people) participated in CHTC pilot from April to September 2011. Of these, 40 couples $(75.5 \%)$ were concordant HIV-negative, three $(5.6 \%)$ were concordant HIV-positive, and ten (18.9\%) were serodiscordant. MSM couples received HTC together. More CHTC programs have been developed since, and they are contributing to increased coverage of HTC among MSM in China.

\subsubsection{Tenofovir in Pregnancy (TIP) Study}

The study entitled "Maternal Tenofovir-Containing Combination Drug Regimen during the Second and Third Trimesters of Pregnancy for Prevention of Mother-toChild Transmission of HIV and HBV in HIV-HBV Co-Infected Mothers" was conducted in Guangxi between 2012 and 2015, in collaboration with the National Center for Women and Children's Health, the local health authorities, and NCAIDS. This phase II clinical trial was primarily supported by the Division of Reproductive Health at US CDC, as well as the Guangxi Bureau of Health and the Guangxi CDC. There was limited information on antiviral therapy for hepatitis B 
virus (HBV) infection among pregnant women coinfected with HIV and HBV (Kourtis et al. 2012). Up to $90 \%$ of infants who acquire their HBV infection perinatally will develop chronic infection. Because HIV/HBV coinfection rates are relatively high among pregnant women in Guangxi province, this study was conducted to provide unique answers to an important research question around prevention of HBV-HIV perinatal transmission to their dually exposed infants (Kourtis et al. 2012; Wang et al. 2016).

\subsubsection{Evaluation of POC CD4 Testing}

CD4 count determination plays an important role in HIV treatment and management decisions. Most conventional CD4 instruments are centrally located in provincial- and prefecture-level facilities with adequate infrastructure and skilled technicians. However, more than $70 \%$ of HIV-infected persons in China live in remote rural areas (Wu et al. 2007). Blood specimens have to be transported to centralized laboratories in a timely and secure manner in order to maintain CD4 cellular integrity. These issues are more prominent in provinces in the West and Southwest of China (e.g., Guangxi, Sichuan, Tibet, Xinjiang, and Yunnan) where a large proportion of patients reside in hard-to-reach, rural, and/or mountainous areas.

A reliable, simple, low-cost, and robust POC device would help overcome these barriers. A joint study on the performance of the Alere PIMATM POC CD4 instrument was carried out in 2012 by the NHRL, Yunnan CDC, Dehong prefecture CDC, and the GAP office in China. In this study, venous and finger-prick blood samples were collected from participants with HIV infection from two VCT sites in Yunnan. Both venous and finger-prick blood specimens were collected from HIV-infected participants. This feasibility study found the PIMA POC CD4 analyzer to be a reasonable alternative to the traditionally employed flow cytometry method (using, e.g., the BD Biosciences FACSCalibur ${ }^{\mathrm{TM}}$ instrument) to determine ART eligibility (Liang et al. 2015). This study found that the Alere PIMA analyzer provides satisfactory CD4 counting using venous blood but is less reliable when finger-prick blood is used. Given the frequent use of phlebotomy in rural China, the POC CD4 analyzer using venous blood could play an important role in improving HIV care in resource-limited settings in China.

\subsection{Capacity Building}

The program has aimed to enhance the national, provincial, and local capacity in response to HIV/AIDS, to strengthen case finding and case management systems.

In May 2004, NCAIDS of China CDC initiated the "Provincial Project Management Training Project (PPMTP)" with the support of GAP. They aimed to strengthen capacity of provincial (and autonomous region and municipality) HIV/ AIDS prevention and control project management staff to improve local project design, implementation, and management. Project management staff responsible for HIV/AIDS prevention and control projects at the provincial level were trained to increase their capacity for project design, planning, implementation, monitoring 
and evaluation, management, and on-the-spot problem-solving. They were also trained to build up knowledge and skills on HIV/AIDS prevention, intervention, treatment, and care. The PPMTP also established a communication platform between provincial CDCs and NCAIDS and a network among provincial CDCs.

This training program invited more than 50 well-known experts and professors on HIV/AIDS prevention and control from universities, NGOs, international organizations, China CDC, NCAIDS, New Century Women's and Children's Hospital (NCWCH) of Beijing, Military Medical Institute, provincial CDCs, infectious disease hospitals, and US CDC to give lectures to trainees. Trainings cover more than 50 topics such as global and China HIV/AIDS epidemics and response strategies, HIV/AIDS counseling, testing and intervention, AIDS clinical treatment and care, HIV virology basic research and testing, project management, and case studies. Post-training evaluation was conducted with the graduates every 2 years. Beginning in 2012, NCAIDS provided funding to support the program directly from the National HIV/AIDS Prevention and Control Fund.

From May 2004 until May 2013, 14 6-month training cohorts were conducted for 112 graduates from 30 provinces and Xinjiang Construction Region and some large cities with independent HIV planning such as Dalian, Qingdao, and Shenzhen. Some graduates have become backbone staff for HIV/AIDS prevention and control in high epidemic areas like Zhumadian prefecture of Henan, Dehong prefecture of Yunnan, Yili prefecture of Xinjiang, and Liuzhou prefecture of Guangxi. Through the training, the professional skills of most graduates increased remarkably. Some graduates were promoted to the position of director or deputy director of HIV/AIDS and other related divisions of their respective CDCs. An evaluation conducted in 2012 showed that $96 \%$ of trainees were satisfied with the program; $77 \%$ were working in planning and managing provincial, national, or international HIV/AIDS programs; $80 \%$ were supervising county-level HIV/AIDS programs; 67\% provided training for others; and $87 \%$ conducted monitoring and evaluation activities.

\subsection{Conclusion}

The first dozen years of strategic technical collaboration of the US CDC GAP in China supported the following: integration with the national and local HIV prevention and control programs; evidence-based decision-making; strengthening systems and capacity at national, provincial, and local levels; prioritizing high-risk geographic areas and populations; developing innovative approaches for scale-up; answering important scientific questions that can be most effectively answered in China but also with global implications for the HIV response; and increasing China's engagement with the global public health community and sharing critical lessons learned.

Domestic funding of China's national HIV program has increased dramatically in recent years (Wu et al. 2011) and promotes more strategic use of scarce resources by targeting a core set of effective interventions (Schwartländer et al. 2011). By targeting key populations for HIV prevention and early initiation of treatment as key 
strategies, new techniques and management concepts were introduced and adapted to the Chinese context. With valuable experiences and momentum, many of these models developed in the first decade of implementation of the GAP and NCAIDS technical collaboration were replicated and expanded nationwide. Chinese technical experts at the GAP office have a unique ability to market the evidence to key stakeholders and to build bridges at provincial and national levels. A productive working relationship with well-conceived models, a results-based activity implementation plan, and proper linkage to the domestic policy process have made significant contributions to further strengthening HIV control and prevention in China.

Acknowledgments I gratefully acknowledge all the GAP staff who have worked tirelessly to make a difference; the US CDC and China CDC staff, especially NCAIDS, AIDS Care China, and other CBOs; and UNAIDS and WHO in China. I would like to especially thank Dr. Zunyou Wu for his special encouragement and support, as well as Dr. Jiangping Sun and other close collaborators at the local, provincial, and national level. Dr. Minghui Ren and Dr. Yu Wang provided leadership support and guidance. Dr. Ray Yip developed and implemented the GAP strategy during the first 4 years of the collaboration. RJ Simonds, Alison Kelly, Chin-Yih Ou, Bharat Parekh, Matt Brown, Jonathan Kaplan, and Colin Shepard made special contributions. Leland (Zhijun) Li and Wendy (Xiaoyu) Wei provided critical management and technical leadership for over a dozen years at the GAP-China office. Button Zhao (now at Global Fund) provided critical epidemiology expertise in the early years. Jennifer McGoogan and Jessica Nan provided input and assisted with editing.

Disclaimer: The findings and conclusions in this report are those of the author and do not necessarily represent the official position of the US Centers for Disease Control and Prevention or the US Department of Health and Human Services.

\section{References}

Bulterys M, Vermund SH, Chen RY, Ou CY. A public health approach to rapid scale-up of free antiretroviral treatment in China: an ounce of prevention is worth a pound of cure. Chin Med J (Engl). 2009;122(11):1352-5.

Chen XS, Wang QQ, Yin YP, Liang GJ, Jiang N, Yang LG, et al. Prevalence of syphilis infection in different tiers of female sex workers in China: implications for surveillance and interventions. BMC Infect Dis. 2012;12:84. https://doi.org/10.1186/1471-2334-12-84.

Chen Y, Bussell SA, Shen Z, Tang Z, Lan G, Zhu Q, et al. Declining inconsistent condom use but increasing HIV and syphilis prevalence among older male clients of female sex workers: analysis from sentinel surveillance sites (2010-2015), Guangxi, China. Medicine (Baltimore). 2016;95:e3726. https://doi.org/10.1097/MD.0000000000003726.

Cheng W, Wu Y, Wen Y, Ma Y, Zhao D, Dou Z, et al. Cotrimoxazole prophylaxis and antiretroviral therapy: an observational cohort study in China. Bull World Health Organ. 2015;93(3):152-60. https://doi.org/10.2471/BLT.14.142745.

Dou Z, Chen RY, Wang Z, Ji G, Peng G, Qiao X, et al. HIV-infected former plasma donors in rural Central China: from infection to survival outcomes, 1985-2008. PLoS One. 2010;5(10):e13737. https://doi.org/10.1371/journal.pone.0013737.

Duan S, Shen S, Bulterys M, Jia Y, Yang Y, Xiang L, et al. Estimation of HIV-1 incidence among five focal populations in Dehong, Yunnan: a hard-hit area along a major drug trafficking route. BMC Public Health. 2010;10:180. https://doi.org/10.1186/1471-2458-10-180.

Fauci AS, Eisinger RW. PEPFAR - 15 years and counting the lives saved. N Engl J Med. 2018;378(4):314-6. https://doi.org/10.1056/NEJMp1714773.

Flannery R. At least 10 million women work in China's sprawling sex trade, author says. Jersey City, NJ: Forbes; 2017. https://www.forbes.com/sites/russellflannery/2017/03/19/ 
at-least-10-million-women-work-in-chinas-sprawling-sex-trade-author-says/\#2b50ebf07374. Accessed 14 Aug 2018.

Han M, Chen Q, Hao Y, Hu Y, Wang D, Gao Y, et al. Design and implementation of a China comprehensive AIDS response programme (China CARES), 2003-2008. Int J Epidemiol. 2010;39(Suppl 2):ii47-55. https://doi.org/10.1093/ije/dyq212.

Han L, Li Z, Luo W, Bulterys M, Yang F, Li R, et al. A qualitative study on reasons for relatively low methadone dosing among persons who inject drugs in three provinces in China. 19th Annual International AIDS Conference, Washington, DC; 2012.

Han L, Zhou C, Li Z, Poon AN, Rou K, Fuller S, et al. Differences in risk behaviours and HIV/STI prevalence between low-fee and medium-fee female sex workers in three provinces in China. Sex Transm Infect. 2016;92(4):309-15. https://doi.org/10.1136/ sextrans-2015-052173.

Jiang Y, Qiu M, Zhang G, Xing W, Xiao Y, Pan P, et al. Quality assurance in the HIV/AIDS laboratory network in China. Int J Epidemiol. 2010;39(Suppl 2):ii72-8. https://doi.org/10.1093/ije/ dyq224.

Kaufman J, Jing J. China and AIDS—-the time to act is now. Science. 2002;296(5566):2339-40. https://doi.org/10.1126/science.1074479.

Kourtis AP, Bulterys M, Hu DJ, Jamieson DJ. HIV-HBV co-infection-a global challenge. N Engl J Med. 2012;366(19):1749-52. https://doi.org/10.1056/NEJMp1201796.

Li J, Wang C, McGoogan JM, Rou K, Bulterys M, Wu Z. Human resource development and capacity building during China's rapid scale-up of methadone maintenance treatment services. Bull World Health Organ. 2013;91(2):130-5. https://doi.org/10.2471/BLT.12.108951.

Liang J, Duan S, Ma YL, Wang JB, Su YZ, Zhang H, et al. Evaluation of PIMA point-of-care CD4 analyzer in Yunnan, China. Chin Med J (Engl). 2015;128(7):890-5.

Lin W, Chen S, Seguy N, Chen Z, Sabin K, Calleja JG, et al. Are HIV surveillance systems adequate in China? Findings from an evaluation of the national HIV/AIDS sentinel surveillance system. Western Pac Surveill Response J. 2012;3(4):76-85. https://doi.org/10.5365/ WPSAR.2012.3.3.004.

Liu E, Rou K, McGoogan JM, Pang L, Cao X, Wang C, et al. Factors associated with mortality of HIV-positive clients receiving methadone maintenance treatment in China. J Infect Dis. 2013;208(3):442-53. https://doi.org/10.1093/infdis/jit163.

Lu F, Wang N, Wu Z, Sun X, Rehnstrom J, Poundstone K, et al. Estimating the number of people at risk for and living with HIV in China in 2005: methods and results. Sex Transm Infect. 2006;82(Suppl 3):iii87-91. https://doi.org/10.1136/sti.2006.020404.

Lu L, Jia M, Ma Y, Yang L, Chen Z, Ho DD, et al. The changing face of HIV in China. Nature. 2008a;455(7213):609-11. https://doi.org/10.1038/455609a.

Lu L, Zhao D, Bao YP, Shi J. Methadone maintenance treatment of heroin abuse in China. Am J Drug Abuse. 2008b;34(2):127-31. https://doi.org/10.1080/00952990701876989.

Ma Y, Zhao D, Yu L, Bulterys M, Robinson ML, Zhao Y, et al. Predictors of virologic failure in HIV-1-infected adults receiving first-line antiretroviral therapy in 8 provinces in China. Clin Infect Dis. 2010;50(2):264-71. https://doi.org/10.1086/649215.

Mi G, Ma B, Kleinman N, Li Z, Fuller S, Bulterys M, et al. Hidden and mobile: a web-based study of migration patterns of men who have sex with men in China. Clin Infect Dis. 2016;62(11):1443-7. https://doi.org/10.1093/cid/ciw167.

Owusu-Edusei K, Tao G, Gift TL, Wang A, Wang L, Yun Y, et al. Cost-effectiveness of integrated routine offering of prenatal HIV and syphilis screening in China. Sex Transm Dis. 2014;41(2):103-10. https://doi.org/10.1097/OLQ.0000000000000085.

Pan S, Li D, Carpiano RM, Spittal PM, Ruan Y. Ethnicity and HIV epidemiology research in China. Lancet. 2016;388(10049):1052-3. https://doi.org/10.1016/S0140-6736(16)31541-0.

Pirkle C, Soundardjee R, Stella A. Female sex workers in China: vectors of disease? Sex Transm Dis. 2007;34(9):695-703. https://doi.org/10.1097/01.olq.0000260989.70866.94.

Poon AN, Li Z, Wang N, Hong Y. Review of HIV and other sexually transmitted infections among female sex workers in China. AIDS Care. 2011;23(Suppl 1):5-25. https://doi.org/10.1080/09 540121.2011.554519. 
Schwartländer B, Stover J, Hallett T, Atun R, Avila C, Gouws E, et al. Towards an improved investment approach for an effective response to HIV/AIDS. Lancet. 2011;377(9782):2031-41. https://doi.org/10.1016/S0140-6736(11)60702-2.

Shan D, Sun J, Khoshnood K, Fu J, Duan S, Jiang C, et al. The impact of comprehensive prevention of mother-to-child HIV transmission in Dehong prefecture, Yunnan Province, 2005-2010: a hard-hit area by HIV in Southern China. Int J STD AIDS. 2014;25(4):253-60. https://doi. org/10.1177/0956462413499010.

Shen T, Csete J. HIV, sex work, and law enforcement in China. Health Hum Rights. 2017;19(2):133-46.

Song J, Feng T, Bulterys M, Zhang D, Korhonen C, Shi X, et al. An integrated city-driven perinatal HIV prevention program covering 1.8 million pregnant women in Shenzhen, China, 2000 to 2010. Sex Transm Dis. 2013;40(4):329-34. https://doi.org/10.1097/ OLQ.0b013e3182805186.

The Lancet. Emerging stronger from the China crisis. Lancet. 2003;361(9366):1311. https://doi. org/10.1016/S0140-6736(03)13083-8.

Wang L, Wang Y, Jin S, Wu Z, Chin DP, Koplan JP, et al. Emergence and control of infectious diseases in China. Lancet. 2008;372(9649):1598-605. https://doi.org/10.1016/ S0140-6736(08)61365-3.

Wang L, Wang N, Wang L, Li D, Jia M, Gao X, et al. The 2007 estimates for people at risk for and living with HIV in China: progress and challenges. J Acquir Immune Defic Syndr. 2009;50(4):414-8. https://doi.org/10.1097/QAI.0b013e3181958530.

Wang N, Wang L, Wu Z, Guo W, Sun X, Poundstone K, et al. Estimating the number of people living with HIV/AIDS in China: 2003-2009. Int J Epidemiol. 2010;39(Suppl 2):ii21-8. https:// doi.org/10.1093/ije/dyq209.

Wang L, Wiener J, Bulterys M, Wei X, Chen L, Liu W, et al. Hepatitis B virus (HBV) load response to 2 antiviral regimens, tenofovir/lamivudine and lamivudine, in HIV/HBV-coinfected pregnant women in Guangxi, China: the Tenofovir in Pregnancy (TiP) Study. J Infect Dis. 2016;214(11):1695-9. https://doi.org/10.1093/infdis/jiw439.

World Health Organization (WHO). Global Health Observatory, Geneva, 2018.

Wu Z, Pisani E. Fulfilling a promise: universal care. In: Wu Z, editor. HIV/AIDS in Chinabeyond the numbers. Singapore: People's Medical Publishing House; 2017. p. 78-99.

Wu Z, Liu Z, Detels R. HIV-1 infection in commercial plasma donors in China. Lancet. 1995;346(8966):61-2.

Wu Z, Rou K, Detels R. Prevalence of HIV infection among former commercial plasma donors in rural eastern China. Health Policy Plan. 2001;16(1):41-6.

Wu Z, Sullivan SG, Wang Y, Rotheram-Borus MJ, Detels R. Evolution of China's response to HIV/ AIDS. Lancet. 2007;369(9562):679-90. https://doi.org/10.1016/S0140-6736(07)60315-8.

Wu Z, Wang Y, Mao Y, Sullivan SG, Juniper N, Bulterys M. The integration of multiple HIV/AIDS projects into a coordinated national programme. Bull World Health Organ. 2011;89(3):227-33. https://doi.org/10.2471/BLT.10.082552.

Wu Z, Xu J, Liu E, Mao Y, Xiao Y, Sun X, et al. HIV and syphilis prevalence among men who have sex with men: a cross-sectional survey of 61 cities in China. Clin Infect Dis. 2013;57(2):298309. https://doi.org/10.1093/cid/cit210.

Wu Z, Wang Y, Detels R, Bulterys M. Towards ending HIV/AIDS among drug users in China (editorial). Addiction. 2015;110(Suppl 1):1-3.

Yang G, Kong L, Zhao W, Wan X, Zhai Y, Chen LC, et al. Emergence of chronic noncommunicable diseases in China. Lancet. 2008;372(9650):1697-705. https://doi.org/10.1016/ S0140-6736(08)61366-5.

Yap L, Wu Z, Liu W, Ming Z, Liang S. A rapid assessment and its implications for a needle social marketing intervention among injection drug users in China. Int J Drug Policy. 2002;13(1):5768. https://doi.org/10.1016/S0955-3959(01)00118-9.

Zhang F, Haberer JE, Wang Y, Zhao Y, Ma Y, Zhao D, et al. The Chinese free antiretroviral treatment program: challenges and responses. AIDS. 2007;21(Suppl 8):S143-8. https://doi. org/10.1097/01.aids.0000304710.10036.2b. 
Zhang F, Dou Z, Yu L, Xu J, Jiao JH, Wang N, et al. The effect of highly active antiretroviral therapy on mortality among HIV-infected former plasma donors in China. Clin Infect Dis. 2008a;47(6):825-33. https://doi.org/10.1086/590945.

Zhang KL, Detels R, Liao S, Cohen M, Yu DB. China's HIV/AIDS epidemic: continuing challenges. Lancet. 2008b;372:1791-3.

Zhang F, Dou Z, Ma Y, Zhao Y, Liu Z, Bulterys M, et al. Five-year outcomes of the China National Free Antiretroviral treatment Program. Ann Intern Med. 2009;151(4):241-51. https://doi. org/10.7326/0003-4819-151-4-200908180-00006.

Zhang F, Dou Z, Ma Y, Zhang Y, Zhao Y, Zhao D, et al. Effect of earlier initiation of antiretroviral treatment and increased treatment coverage on HIV-related mortality in China: a national observational cohort study. Lancet Infect Dis. 2011;11(7):516-24. https://doi.org/10.1016/ S1473-3099(11)70097-4.

Zhang L, Chow EP, Jing J, Zhuang X, Li X, He M, et al. HIV prevalence in China: integration of surveillance data and a systematic review. Lancet Infect Dis. 2013;13(11):955-63. https://doi. org/10.1016/S1473-3099(13)70245-7.

Zhang F, Zhu H, Wu Y, Dou Z, Zhang Y, Kleinman N, et al. HIV, hepatitis B virus, and hepatitis $\mathrm{C}$ virus co-infection in patients in the China National Free Antiretroviral Treatment Program, 2010-2012: a retrospective observational cohort study. Lancet Infect Dis. 2014;14(11):106572. https://doi.org/10.1016/S1473-3099(14)70946-6.

Zhu Q, Wang L, Lin W, Bulterys M, Yang W, Sun D, et al. Improved survival with cotrimoxazole prophylaxis among people living with HIV/AIDS who initiated antiretroviral treatment in Henan Province, China. Curr HIV Res. 2014;12(5):359-65. https://doi.org/10.2174/15701 $62 X 1205141121102155$. 